\title{
Candidate of metastasis 1 regulates in vitro growth and invasion of bladder cancer cells
}

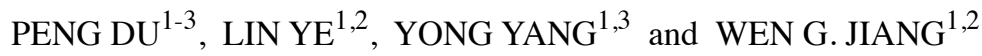 \\ ${ }^{1}$ Cardiff University-Peking University School of Oncology Joint Institute, ${ }^{2}$ Metastasis and Angiogenesis Research Group, \\ Cardiff University School of Medicine, Heath Park, Cardiff CF14 4XN, UK; ${ }^{3}$ Key Laboratory of Carcinogenesis \\ and Translational Research (Ministry of Education), Department of Urology, \\ Peking University Cancer Hospital, Haidian District, Beijing, P.R. China
}

Received July 2, 2012; Accepted August 29, 2012

DOI: $10.3892 /$ ijo.2013.1802

\begin{abstract}
COM1 (candidate of metastasis 1) has been recently shown to influence the metastatic ability of cancer cells and disease progression of certain solid tumours. The role of COM1 in bladder cancer remains unknown. In the present study, we examined the expression of the COM1 protein in human bladder tissues, and also its effect on growth, adhesion, migration and invasion of human bladder cancer cells, in vitro. The expression of COM1 in human bladder tissues and bladder cancer cell lines was assessed at both the mRNA and protein levels using RT-PCR and immunohistochemistry, respectively. COM1 staining was compared with tumour staging. Mammalian COM1 expression construct and anti-COM1 ribozyme transgenes were used to generate sublines of human bladder cancer cells with differential expression of COM1. The effect of COM1 on cellular functions was examined in bladder cancer cells with which COM1 was overexpressed or knocked down using a variety of in vitro assays. In normal bladder tissues, stronger staining of COM1 was seen in the cytoplasm of normal urothelial cells. In contrast, the staining was notably weak or absent in cancer cells of tumour tissues and invasive tumours had significantly low levels of staining compared with non-invasive tumours $(p=0.012)$. Knockdown of COM1 in bladder cancer cell lines resulted in an increase in cellular growth and invasion, while overexpression of COM1 suppressed invasiveness and growth of these cells. Further investigation revealed an increased apoptosis and upregulated p21 in bladder cancer cells when COM1 was overexpressed. COM1 is expressed at low levels in human
\end{abstract}

Correspondence to: Professor Yong Yang, Department of Urology, Peking University Cancer Hospital, Fucheng Road, Haidian District, Beijing, P.R. China

E-mail: yoya_urology@sina.com

Professor Wen G. Jiang, Metastasis and Angiogenesis Research Group, Cardiff University School of Medicine, Cardiff CF14 4XN, UK

E-mail: jiangw@cf.ac.uk

Key words: candidate of metastasis-1, p8, bladder cancer, growth, invasion, p21, apoptosis bladder cancer and in particular in invasive bladder tumours. COM1 levels are inversely correlated with the invasiveness and growth of bladder cancer cells in vitro. Induced apoptosis and upregulation of p21 are indicated in the mechanism of COM1 inhibiting bladder cancer cell growth. It suggests that COM1 is a potential tumour suppressor in human bladder cancer.

\section{Introduction}

COM1 (candidate of metastasis 1) was initially found to be upregulated in the metastatic lesions of the central nervous system upon injection of cancer cells originally isolated from micrometastases in the bone marrow of a breast cancer patient (1). Subsequently COM1 was found to be the human counterpart of the rat $p 8$, which was first reported as a new gene and strongly activated in pancreatic acinar cells during the acute phase of pancreatitis in rat described by Mallo et al (2). COM1/P8 also known as nuclear protein transcriptional regulator 1 (NUPRl), is located on chromosome 16 at position p11.2. The COM1 gene encodes two isoforms in protein products, one with 100 amino acids, the other with 82 amino acids. The role of the 18 amino acids region absent in the latter is presently unclear.

Overexpression of $C O M 1$ has been indicated in the pancreatic cancer and thyroid cancer $(3,4)$. Su et al reported that COM1 was overexpressed in $59 \%$ of the 44 pancreatic cancer cohort. In addition, its expression was inversely correlated to apoptosis in pancreatic cancer. However, COM1 did not have any significant correlation with the overall survival of the patients with pancreatic cancer. Moreover, COM1 was more prominent in lower age group patients, moderately or poorly differentiated cancers and lymph node positive cases. Overexpression of COM1 and loss of apoptosis were significantly correlated with poor differentiation and lymph node metastasis. This interesting expression pattern might reflect not only the growth activity and differentiation of cancer cells but also invasion and metastasis of human pancreatic cancer (3). Ito et al reported that COM1 was significantly overexpressed in thyroid papillary carcinomas and that COM1 overexpression was more frequently observed in larger papillary carcinoma with lymph node metastasis (4).

On the other hand, reduced expression of COM1 was revealed in other cancer types including breast and prostate 
Table I. Primer sequences for PCR.

\begin{tabular}{lll}
\hline Primer & \multicolumn{1}{c}{ Forward } & \multicolumn{1}{c}{ Reverse } \\
\hline COM1 & CCTGGATGAATCTGACCTC & AGCAGCTTCTCTCTTGGTG \\
COM1 expression & ATGGCCACCTTCCCAC & ACTGCGCCGTGCCCCTCG \\
COM1 ribozyme & CTGCAGCTTCTCTCTTGGTGCCTGATGAGTCC & ACTAGTGGAGGCCGGAAAGGTTTCGTCC \\
& GTGAGGA & TCACGGACT \\
uPA & GGTTGTGTGTGGGTTAGACT & GTGGCTACCAGACATTGATT \\
p21 & CGGGATGAGTTGGGAGGAG & ACAGGTCCACATGGTCTTCC \\
C-myc & TGCTCCATGAGGAGACAC & TTTCATTGTTTTCCAACTCC \\
GAPDH & GGCTGCTTTTAACTCTGGTA & GACTGCGGTCATGAGGCCGT \\
\hline
\end{tabular}

cancer. Breast cancer cells have markedly reduced nuclear distribution of the COM1 protein compared with normal mammary epithelial cells. Low levels of COM1 were associated with nodal status and poor clinical outcome (5). A follow-up study showed that the nuclear presence of the COM1 protein was crucial to its inhibitory role in cancer cells, and that loss of its nuclear presence of this molecule had a detrimental effect on its overall negative control of cancer growth (6). Compared with normal prostate tissues, the expression of COM1 in prostate cancer cells was substantially reduced. Knockdown of COM1 in prostate cancer cells resulted in increased cellular motility and growth. In contrast, overexpression of COM1 suppressed invasiveness and growth of prostate cancer cells and tumour growth in vivo. COM1 was proposed to act as a potential tumour suppressor in prostate cancer (7). Collectively these studies suggest that $C O M 1$ plays contrasting roles in different malignancies. Although the mode of action of COM1 in cancer cells remains unclear, it has been recently indicated that Com1 may be connected to doxobubicin-indued chemoresistence in cancer cells and that this may be linked to the regulation of p53 and p21 (8).

Although COM1 has been implicated in the disease progression of certain solid tumours including prostate cancer, its role in bladder cancer remains unknown. In the present study we first examined the expression of COM1 in normal bladder tissues and bladder tissues with different invasion status and the effect of COM1 on growth, adhesion, migration and invasion of bladder cancer cells.

\section{Materials and methods}

Materials, cell lines and tissue samples. Human bladder cancer cell lines RT112, EJ138 and T24 (ECACC, European Collection of Animal Cell Culture, Salisbury, UK) were routinely maintained in DMEM-F12 medium supplemented with $10 \%$ fetal bovine serum and antibiotics. Polyclonal goat anti-COM1 (SC-23283) and monoclonal mouse anti-GAPDH (SC-32233) were obtained from Santa Cruz Biotechnology (Santa Cruz, CA, USA). Other reagents or kits were obtained from Sigma-Aldrich, Poole, UK.

Bladder tissues samples ( $n=37$, including 14 non-invasive, 18 invasive and 5 normal bladder tissues) were obtained from the Department of Urology, Chaoyang Hospital, Beijing, China. All protocols were reviewed and approved by the Ethics Committee and all patients gave written informed consent.
Immunohistochemical staining procedure for bladder tissues. Thin paraffin tissues sections were first dewaxed using the gradient xylene/ethanol solutions. Antigen retrieval was carried out using an EDTA antigen retrieving buffer in microwave (25 min). After blocking the endogenous peroxidase, the sections were incubated for $20 \mathrm{~min}$ in a horse serum blocking solution and probed with the primary antibody $(1 \mathrm{~h})$. Following extensive washing, sections were incubated for 30 min with a biotinylated secondary antibody (multilink swine anti-goat/mouse/rabbit immunoglobulin, Dako Inc. Carpinteria, CA, USA). Following washing, an avidin-biotin complex (Vector Laboratories) was applied to the sections followed by extensive washing. Diamino benzidine chromogen (Vector Laboratories) was then added to the sections, which were incubated in the dark for $5 \mathrm{~min}$. Sections were then counterstained in Gill's haematoxylin and dehydrated in ascending grades of methanol before clearing in xylene and mounting under a cover slip. Staining of COM1 was assessed using a method modified from Allred IHC scoring system (9). Score $0,1,2$ and 3 was given to the staining being negative, faint, moderate and strong, respectively.

Construction of COM1 expression vectors and transfection. The first strand cDNA was synthesized from RNA isolated from normal human mammary tissues using a DuraScript ${ }^{\mathrm{TM}}$ RT-PCR kit. PCR was then used to amplify the coding sequence of human COM1 using the Extensor Hi-Fidelity PCR Master mix (ABgene Ltd., Epsom, UK). The sequences of primers are shown in Table I. The verified COM1 insert was cloned into a mammalian expression plasmid vector $(\mathrm{pEF} / \mathrm{His}$ TOPO TA plasmid vector, Invitrogen Inc., Paisley, UK). The recombinant plasmid vectors were transformed into chemically competent TOP10 E. coli (Invitrogen), and the colonies were then analyzed. Colonies carrying correct recombinant plasmids were amplified and plasmids extracted. Purified COM1 transgenes and control plasmid vectors were then transfected into RT112 and EJ138 cells individually using an Easjet Plus electroporator (EquiBio Ltd, Kent, UK). After up to 3 weeks of selection with blasticidin the transfectants were verified for their expression of COM1 and successful clones were used in subsequent studies.

Generation of COM1 ribozyme transgenes. Anti-COM1 hammerhead ribozyme transgenes were synthesised and cloned into $\mathrm{pEF6/V5-His-TOPO} \mathrm{plasmid} \mathrm{vector} \mathrm{as} \mathrm{described} \mathrm{in}$ 
Table II. IHC staining of COM1 in bladder cancer specimens.

\begin{tabular}{llccccr}
\hline & & Score 0 $(\%)$ & Score 1 $(\%)$ & Score 2 (\%) & Score 3 (\%) & Total \\
\hline Normal & & 0 & $2(40)$ & $3(60)$ & 0 & 5 \\
Cancer & Non-invasive & 0 & $2(14.3)$ & $9(64.3)$ & $3(21.4)$ & 14 \\
& Invasive & $3(16.7)$ & $10(55.6)$ & $4(22.2)$ & $1(5.6)$ & 18 \\
\hline
\end{tabular}

Score 0 , negative; score 1, faint staining; score 2, moderate staining; score 3, strong staining. COM1 staining was decreased in invasive bladder cancer, $\mathrm{p}=0.047 \mathrm{in}$ comparison with its staining in normal bladder tissues and non-invasive bladder cancer using $\chi^{2}$ test. Invasive tumours had a significantly low level of straining compared with non-invasive tumours $\left(\mathrm{p}=0.012, \chi^{2}=10.9\right)$.

previous studies (5-7). Empty plasmid vectors and the ribozyme transgenes were then transfected into RT112 and EJ138 cells and the cells underwent selection with $5 \mu \mathrm{g} / \mathrm{ml}$ blasticidin for approximately 2 weeks before verification.

RNA isolation and reverse transcription PCR. RNA was isolated using total-RNA Isolation Reagent (ABgene Ltd). Reverse transcription was performed using the DuraScript ${ }^{\mathrm{TM}}$ RT-PCR kit, followed by PCR using a REDTaq ${ }^{\mathrm{TM}}$ ReadyMix PCR reaction mix (primer sequences shown in Table I). Cycling conditions were $94^{\circ} \mathrm{C}$ for $5 \mathrm{~min}$, followed by 30 cycles of $94^{\circ} \mathrm{C}$ for $30 \mathrm{sec}, 55^{\circ} \mathrm{C}$ for $30 \mathrm{sec}$ and $72^{\circ} \mathrm{C}$ for $40 \mathrm{sec}$. This was followed by a final $10-\mathrm{min}$ extension period at $72^{\circ} \mathrm{C}$. The products were visualized on $2 \%$ agarose gel stained with ethidium bromide.

Immunoprecipitation and western blot analysis. The protein concentration in cell lysates were determined using the DC Protein Assay kit (Bio-Rad) and an ELx800 spectrophotometer $\left(\right.$ Bio-Tek $\left.^{\mathrm{TM}}\right)$. Equal amount of proteins were separated by sodium dodecyl sulfate-polyacrylamide gel electrophoresis (SDS-PAGE) and blotted onto nitrocellulose sheets. Proteins were then respectively probed with anti-COM1 antibody and peroxidase-conjugated secondary antibody, with stringent washings between each step. Protein bands were visualized using the Supersignal ${ }^{\mathrm{TM}}$ West Dura system (Pierce Biotechnology, Inc., Rockford, IL, USA), and photographed using a UVITech imager (UVITech Inc., Cambridge, UK).

In vitro cell growth assay. Cells were plated into a 96-well plate (3,000 cells/well). Cell growth was assessed after a period of incubation (up to 5 days). Crystal violet was used to stain cells. Following washing, stained crystal was extracted with $10 \%$ acetic acid and absorbance was determined at a wavelength of $540 \mathrm{~nm}$ using a spectrophotometer (Elx800, Bio-Tek, Potton, UK).

In vitro invasion assay. Transwell inserts with $8 \mu \mathrm{m}$ pore size were coated with $50 \mu \mathrm{g}$ Matrigel (BD Matrigel ${ }^{\mathrm{TM}}$ Basement Membrane Matrix) and air dried. The Matrigel was rehydrated before use. Cells $(30,000)$ were added to each insert, same number cells were loaded into another well as control. After $96 \mathrm{~h}$ cells that had migrated through the matrix to the other side of the insert were fixed in $4 \%$ formalin, stained with $0.5 \%$ $(\mathrm{w} / \mathrm{v})$ crystal violet. The stained crystal was extracted with $10 \%$ acetic acid and absorbance was determined at a wavelength of $540 \mathrm{~nm}$ using a spectrophotometer (Bio-Tek, Elx800). Percentage of invaded cells was calculated as absorbance of invaded cells divided by absorbance of control cells.

Flow cytometric analysis of apoptosis. All cells including those floating in the culture medium were harvested after a period of incubation. Cells were washed in ice cold PBS and resuspended in $1 X$ Annexin-binding buffer at a density of $1 \times 10^{6}$ cells $/ \mathrm{ml}$ after centrifugation. FITC Annexin V $(5 \mu \mathrm{l})$ and PI working solution (1 $\mu \mathrm{l})(100 \mu \mathrm{g} / \mathrm{ml})$ (Molecular Probes, Eugene, OR, USA) were added to $100 \mu \mathrm{l}$ of the cell suspension. After a 30-min incubation at room temperature, $400 \mu \mathrm{l}$ of $1 \mathrm{X}$ Annexin-binding buffer was added, mixed gently and the samples were kept on ice. The stained cells were immediately analyzed using the flow cytometer and FlowMax software package.

Statistical analysis. Statistical analysis was performed using the Minitab statistical software package (version 14). Non-normally distributed data were assessed using the Mann-Whitney test, while the two sample t-test was used for normally distributed data. $\chi^{2}$ test was used to analyse the IHC staining of COM1 in bladder specimens. Differences were considered to be statistically significant at $\mathrm{p}<0.05$.

\section{Results}

Expression of COM1 in human bladder tissues. We first examined the protein expression of COM1 in human bladder tissues using immunohistochemical method. As shown in Fig. 1A, staining of COM1 was mainly confined in cytoplasm of bladder urothelial cells, with no obvious staining seen in nuclei of these cells or stromal tissue. Normal bladder urothelial cells and cancer cells of non-invasive tumours exhibited a stronger staining of COM1 in the cytoplasm. In contrast, the staining was weaker or absent from the cancer cells of invasive tumour tissues (Fig. 1A). Invasive tumours had significantly low levels of staining in comparison with non-invasive tumours $(\mathrm{p}=0.012$, Table II). In both normal and tumour tissues, nucleic staining of COM1 was not demonstrated. The expression of COM1 was also examined in three bladder cancer cell lines using RT-PCR. COM1 transcript was detected in all three human bladder cancer cells (Fig. 1B).

Knockdown and overexpression of COM1 in bladder cancer cells. To investigate the impact of COM1 on functions of bladder cancer cells, the constructed COM1 expression vectors 

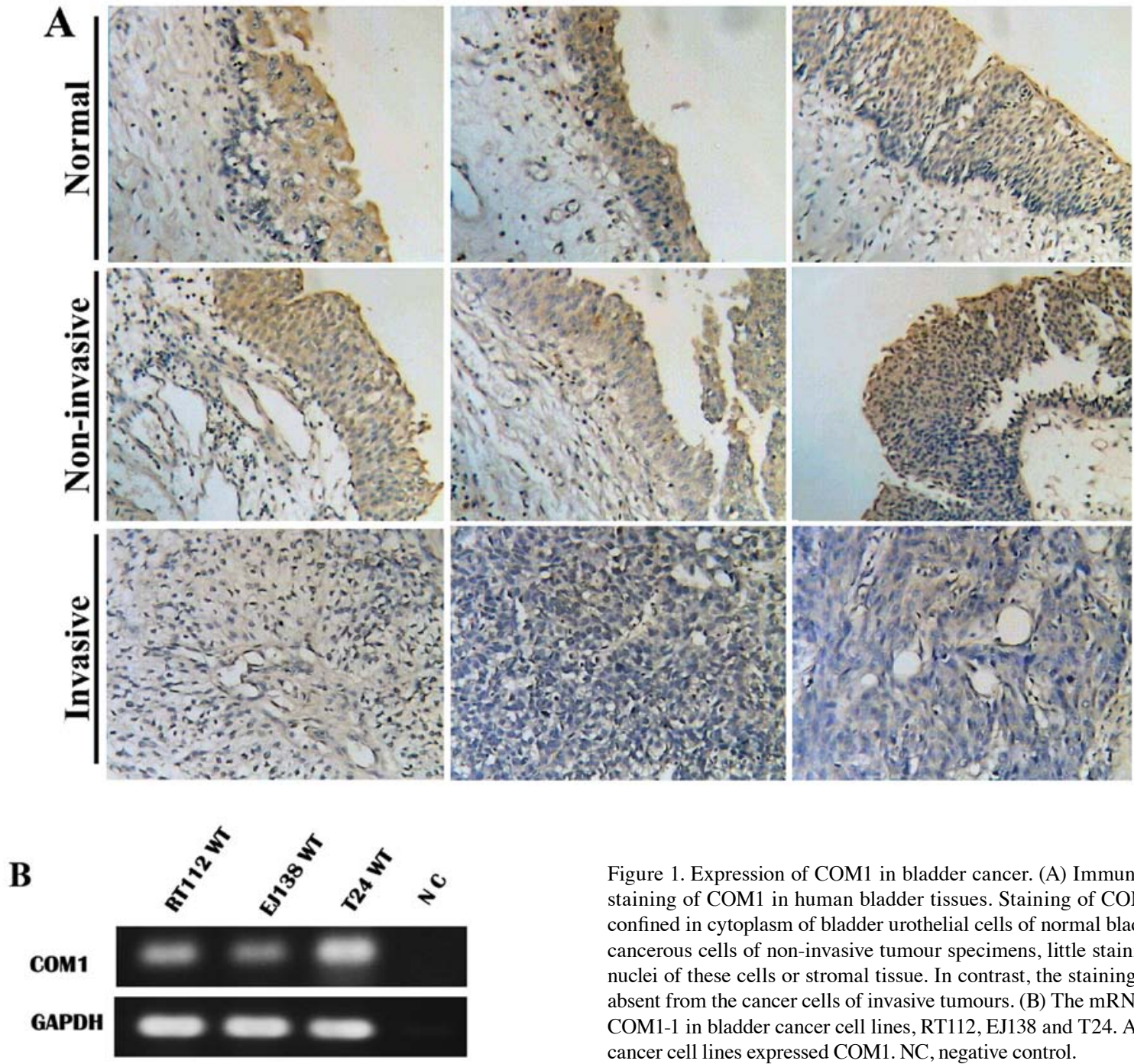

Figure 1. Expression of COM1 in bladder cancer. (A) Immunohistochemical staining of COM1 in human bladder tissues. Staining of COM1 was mainly confined in cytoplasm of bladder urothelial cells of normal bladder tissues and cancerous cells of non-invasive tumour specimens, little staining was seen in nuclei of these cells or stromal tissue. In contrast, the staining was weaker or absent from the cancer cells of invasive tumours. (B) The mRNA expression of COM1-1 in bladder cancer cell lines, RT112, EJ138 and T24. All three bladder cancer cell lines expressed COM1. NC, negative control.

and anti-COM1 ribozyme transgenes were utilised to overexpress or knockdown COM1 in bladder cancer cells, which were weakly positive for COM1. After the selection using blasticidin, the expression of COM1 in the transfected cells was verified using both RT-PCR and western blot analysis (Fig. 2). Reduced COM1 expression of both mRNA (Fig. 2A) and protein (Fig. 2B) was seen in RT112 COM1rib, in comparison with RT112 WT and RT112 pEF, while an increased expression of COM1 was seen in RT112 COMexp compared to the controls. Similarly, knockdown and overexpression of COM1 were confirmed in EJ138 COMrib and EJ138 COMexp cells respectively, in comparison with EJ138 WT and EJ138 pEF control cells.

COM1 is associated with the growth rate and invasion of bladder cancer cells. Following verification, influence of COM1 on invasiveness was examined in the genetically modified cells. Knockdown of COM1 in the bladder cancer cells rendered the cells more invasive (Fig. 3). Interestingly, overexpression of COM1 in these cells made the cells less invasive compared with the control cells. Using the same cells, we further tested the rate of cell growth over a 5-day period. RT112 COM1rib and EJ138 COM1rib both displayed a faster rate of growth, compared with the controls (Fig. 4).
Furthermore, cells with forced overexpression of COM1 displayed a slower growth rate.

Effects of COMI on apoptosis of bladder cancer cells. In order to determine the mechanism by which COM1 inhibits bladder cancer cell growth, the proportion of apoptotic cells was analyzed by flow cytometry. It was demonstrated that there was a significantly higher proportion of apoptotic cells including both early apoptotic (Q4), late apoptotic and dead cells $(\mathrm{Q} 2)$ in RT112 COM1exp cells (Q2 9.77\% + Q4 15.64\%), compared with the RT112 WT (Q2 7.58\% + Q4 9.65\%) and the RT112pEF (Q2 7.75\% + Q4 8.36\%) control (Fig. 5). There was no difference of the apoptotic population was seen in RT112COM1rib cells compared to both controls.

Expression of uPA, C-myc and P21 in bladder cancer cell line RT112. We further examined a couple of COM1 responsive genes which might be involved in the impact on growth and invasion. The mRNA expression of uPA, C-myc and P21 in RT112 cells were detected using RT-PCR. The expression of P21 was significantly higher in which bladder cancer cells that overexpressed COM1, and was much lower in COM1 knockdown cells. But no significant difference was found in mRNA levels of uPA and C-myc (Fig. 6). 

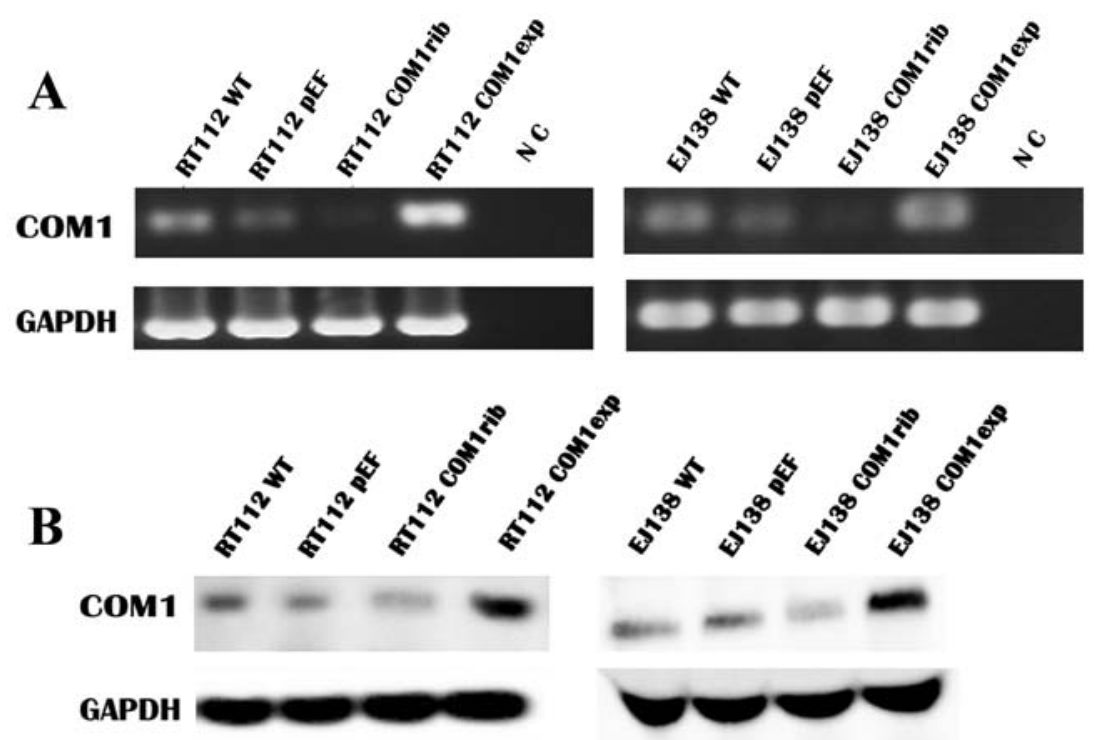

Figure 2. Knockdown and overexpression of COM1 in bladder cancer cells. (A) The mRNA expression of COM1 in the transfected cells by RT-PCR. mRNA of COM1 was knocked down in RT112 COM1rib and EJ138 COM1rib, and overexpressed in RT112 COM1exp, compared with the wild-type (RT112WT and EJ138WT) and empty plasmid (RT112pEF and EJ138pEF) control cells. Reduced COM1 expression of mRNA was seen in RT112 COM1rib, in comparison with RT112 WT and RT112 pEF, while an increased expression of COM1 was seen in RT112 COMexp compared to the controls. Similarly, knockdown and overexpression of COM1 were confirmed in EJ138 COMrib and EJ138 COMexp cells respectively, in comparison with EJ138 WT and EJ138 pEF control cells. NC, negative control. (B) The potein expression of COM1 $(\sim 10 \mathrm{kDa})$ in the transfected cells by western blot analysis. Protein of COM1 was knocked down in RT112 COM1rib and EJ138 COM1rib and overexpression in RT112 COM1exp, compared with the wild-type (RT112WT and EJ138WT) and empty plasmid (RT112pEF and EJ138pEF) control cells. Reduced COM1 expression of protein was seen in RT112 COM1rib, in comparison with RT112 WT and RT112 pEF, while an increased expression of COM1 was seen in RT112 COMexp compared to the controls. Similarly, knockdown and overexpression of COM1 were confirmed in EJ138 COMrib and EJ138 COMexp cells respectively, in comparison with EJ138 WT and EJ138 pEF control cells. GAPDH (38 kDa) was used as the housekeeping control.
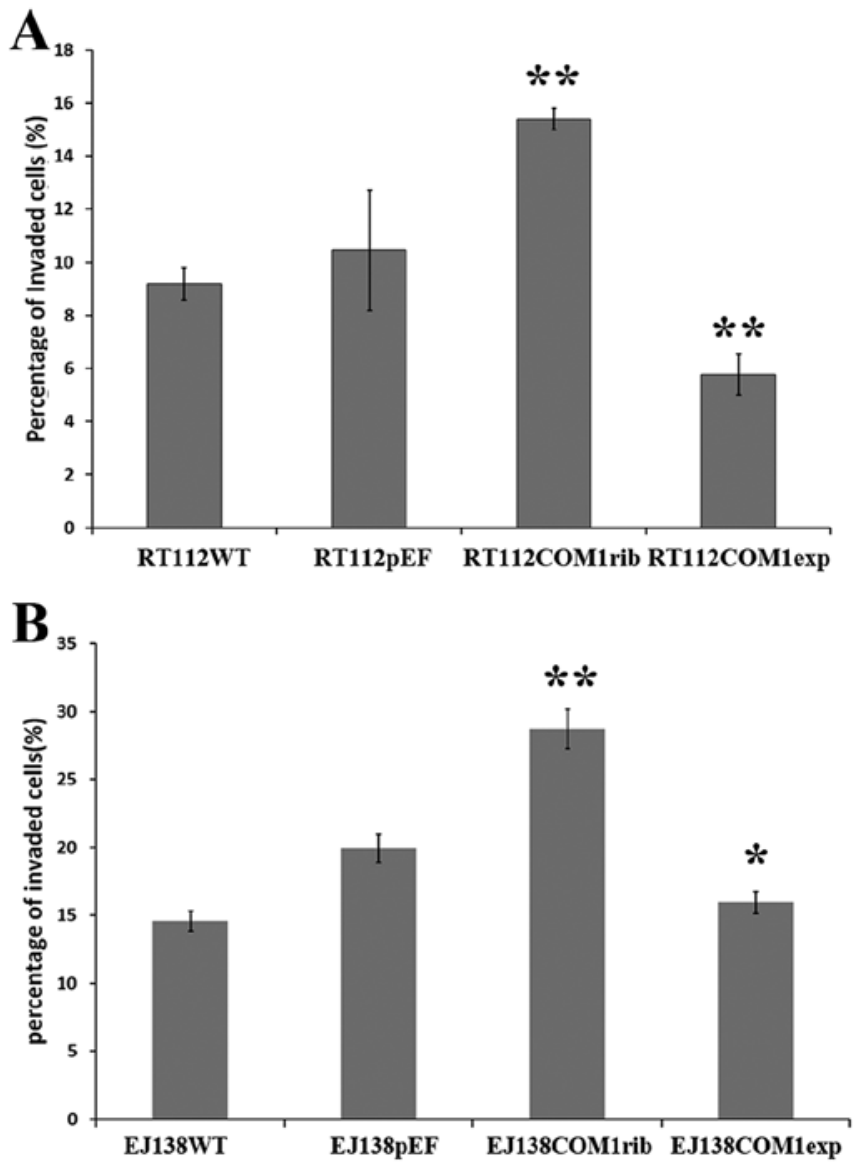

Figure 3. Effects of COM1 knockdown and overexpression on the invasion, in vitro. (A) RT112 cells, (B) EJ138 cells. "p<0.05 versus the respective controls, ${ }^{* *} \mathrm{p}<0.01$ versus the controls. The experiment was repeated three times.
A

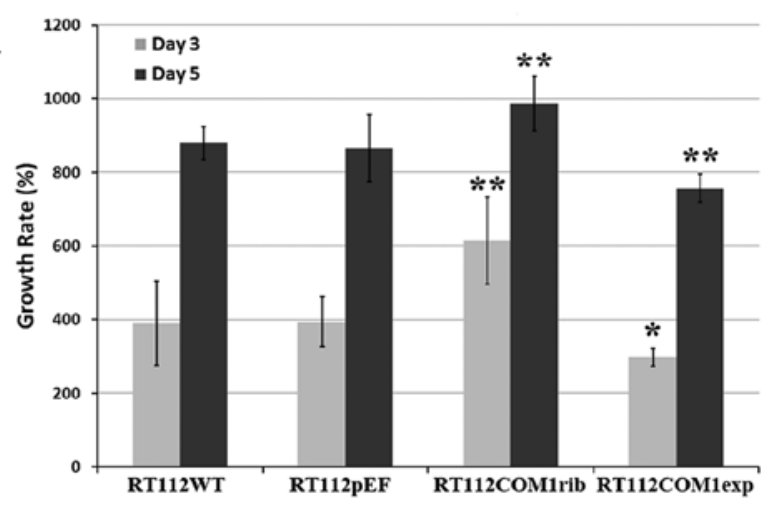

B

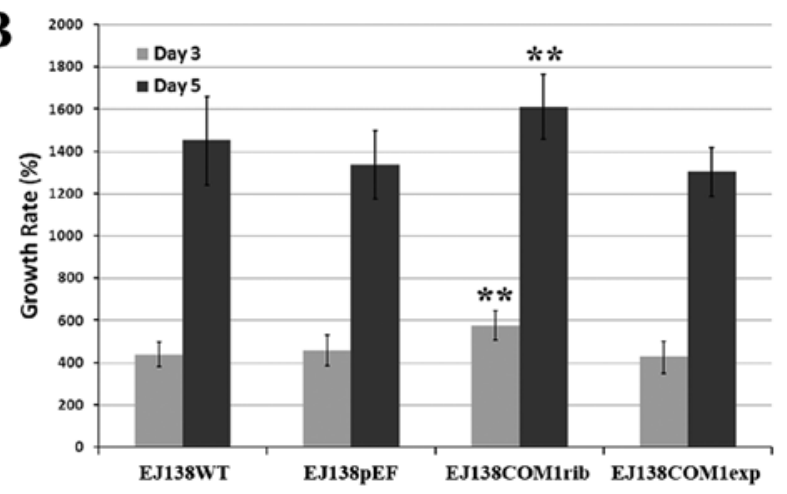

Figure 4. Effect of COM1 expression on growth rate of bladder cancer cells. (A) The cell growth rate (shown are third day and fifth day) of RT112 COM1rib cells was significantly increased in comparison with the controls (WT and pEF, $\mathrm{n}=12),{ }^{* *} \mathrm{p}<0.01$. The cell growth rate of RT112 COM1exp was significantly reduced compared to both controls, ${ }^{* *} \mathrm{p}<0.01$ for RT112 COM1exp versus RT112 WT, and $\mathrm{p}<0.001$ versus RT112 pEF $(n=12)$. (B) The cell growth rate of EJ138 COM1rib cells was significantly increased in comparison to both WT and $\mathrm{pEF}$, ${ }^{*} \mathrm{p}<0.01$. The experiment was repeated more than three times. 


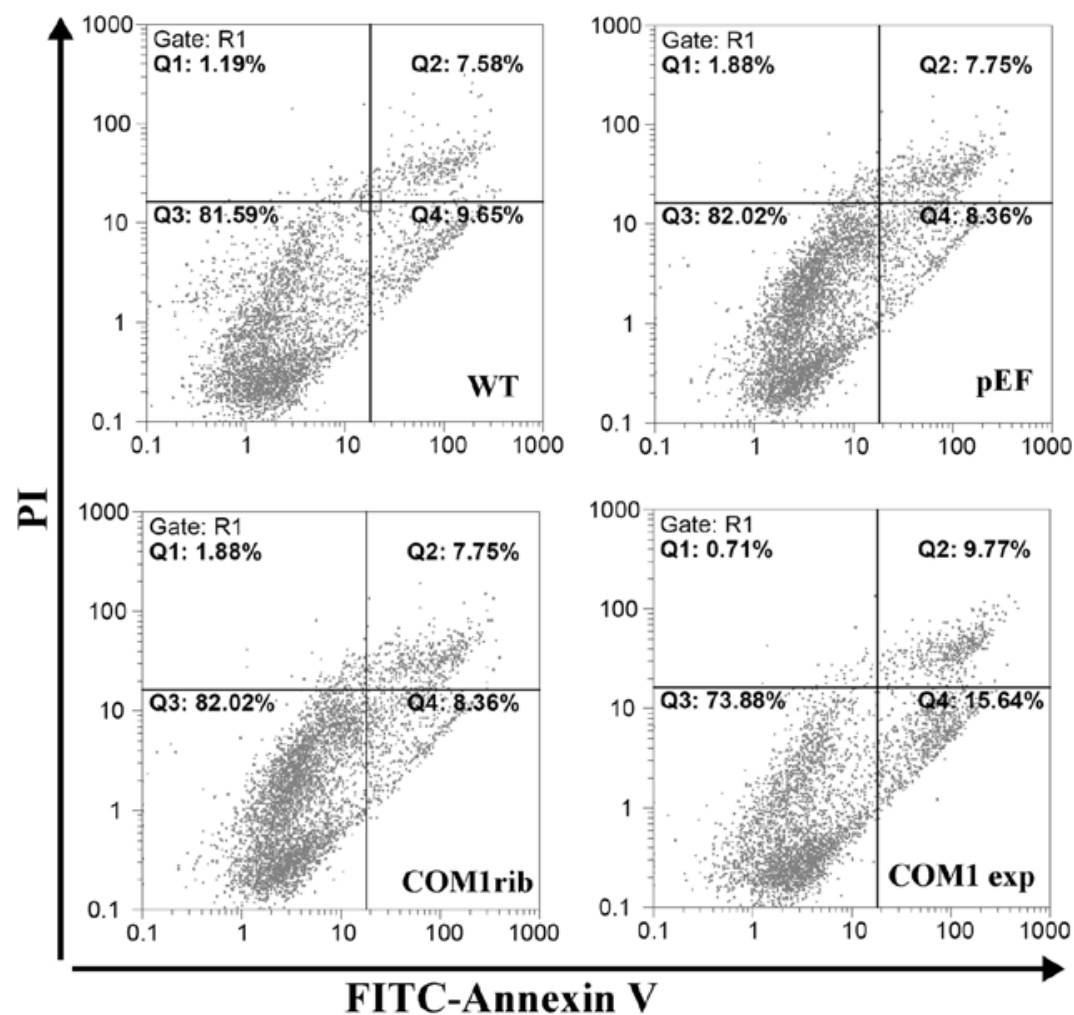

Figure 5. The apoptotic population in RT112 cells by flow cytometry. The apoptotic population in RT112 cells was analyzed using flow cytometry after $48 \mathrm{~h}$ of serum starvation. There was a marked increase in the population of apoptotic cells in the RT112 COMlexp cells compared to the controls. No significant decrease in the numbers was observed of apoptotic cells in RT112 COM1rib cells compared to the controls (WT and pEF). The experiment was repeated three times. Apoptotic index refers to total apoptotic population including both early apoptotic cells (Q2) and late apoptotic cells (Q4).

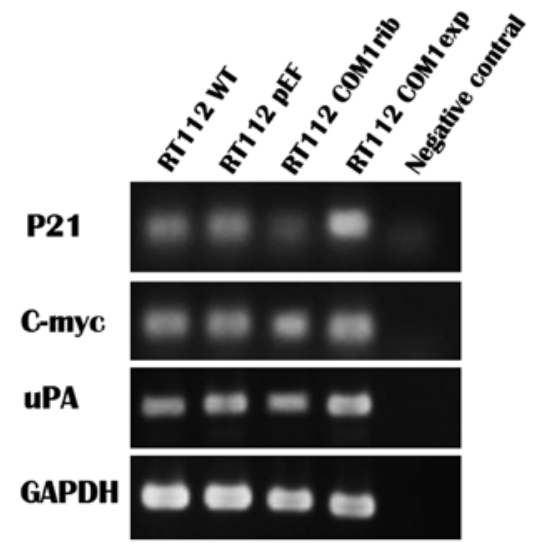

Figure 6. Expression of uPA, C-myc and P21 in bladder cancer cell line RT112 in relation with COM1. The mRNA expression of uPA, C-myc and P21 in RT112 cells were detected using RT-PCR. The expression of P21 was significantly higher in bladder cancer cells that overexpressed COM1 and was much lower in COM1 knockdown cells. No significant difference was found in mRNA levels of uPA and C-myc.

\section{Discussion}

Bladder cancer is one of the commonly seen tumours in males and females and the incidence is estimated to be the 7th in the UK and 9th worldwide, based on the 2008 statistics (Cancer Research UK, 2012) Approximately 382,660 new bladder cancer cases occurred and 150,280 patients died of bladder cancer worldwide in 2008 . At the same time, bladder cancer is the most common malignancy of the urinary tract, accounting for $90-95 \%$ of urothelial carcinomas (11). The feature of bladder cancer is apt to recurrence. At initial diagnosis, $75 \%$ patients present with non-muscle-invasive bladder cancer (NMIBC), the remaining $25 \%$ present with muscle-invasive bladder cancer (MIBC). The main problems of NMIBC are recurrence and progression, while MIBC is frequently associated with metastatic diseases and is the major cause of mortality. In NMIBC, approximately $50-80 \%$ of patients will recur after managed with transurethral resection (TUR) and intraversical therapy (12). Despite the improvements in both early detection and treatment, there remains a significant challenge to manage this disease.

The role of COM1, in tumour progression remains unclear and in somewhat controversial. However, its effect on cell growth, i.e. whether it is stimulating or inhibiting may vary in different malignancies. COM1 has been found to aid the establishment of metastasis and to play a key role in the progression of malignancies of breast $(1,5-7,13)$, thyroid $(4,13,14)$, central nervous system $(15)$ and pancreas $(3,16,17)$. COM1 has been implicated in inducing chemoresistance in pancreatic and breast cancer cells, protecting them from apoptosis and making tumour cells genetically unstable (17). In prostate cancer, however, COM1 appears to have tumour suppressive activity (7). The current study has examined the staining pattern of COM1 in human bladder tissues and tested the impact on the growth, invasion and apoptosis of bladder cancer cells by genetically manipulating the expression of COM1.

Similar to human breast cancer, thyroid and prostate cancer, COM1 is seen at a lower level in bladder tumour cells, compared 
with normal bladder urothelial cells and the COM1 staining is linked to the muscle invasion of bladder cancer. This is in contrast to that observed with pancreatic cancer cells, in which cancer cells had higher staining than normal cells. This would indicate that in solid human tumours, COM1 exists at lower levels, although the opposite may also operate. It is interesting to note that both normal urothelial cells and bladder cancer cells had little COM1 in the nucleus. The nuclear existence of COM1 is particularly interesting as it has been suggested that the cytoplasmic/nuclear distribution pattern of the COM1 protein may be a key feature in cancer and an important reason in deciding the contrasting role of COM1 in different cancer types. While COM1 nucleic staining in breast cancer cells has been shown to be significantly reduced compared with normal epithelial cells (5), bladder cancer cells have a marked reduction in the cytoplasmic region while no nuclear staining was seen in normal and tumour cells of the bladder tissues. The current study further indicates the possibility of a nuclear connection in the function of COM1. Thus, changes in the overall level of staining of COM1 in bladder cancer cells and in intracellular distribution appear to be a feature in human bladder tumour tissues.

In the present study, we employed methods to genetically alter the expression of COM1 in bladder cancer cells, namely the ribozyme approach and overexpression approach, respectively. The loss of COM1 by way of hammerhead ribozyme transgenes resulted in more invasiveness compared with control cells. In clear contrast, forced overexpression of COM1 in two bladder cancer cell lines, RT112 and EJ138, resulted in a reduction of invasion. This indicates that COM1 plays a key role in the control of the aggressiveness of bladder cancer cells. The current study has also demonstrated that loss of COM1 resulted in an accelerated cell growth in vitro. This growth regulatory role of COM1 has been tentatively indicated in other tumour cells including breast cancer cells and thyroid cancer cells, in which Vit D3 inhibition of cell growth was associated with a rise of COM1 $(18,19)$. In pancreatic cancer cells, overexpression of COM1 is associated with inhibitory effects on cell growth and/or with accelerated apoptosis (15). This together with the effects observed in other solid tumours (breast, prostate and thyroid) $(4,6,14)$, strongly suggest that COM1 is a potential tumour suppressor in these solid tumours. This suggestion is further supported by our in vitro results, in which COM1 exhibited inhibitory effect on growth of bladder cancer cells.

In order to determine the mechanism by which COM1 inhibits bladder cancer cell growth, the proportion of apoptotic cells was analyzed by flow cytometry in our study. There was an increased proportion of apoptotic cells (both early and late) in bladder cancer cells overexpressing COM1. A cyclin/cyclin dependent kinase (cdk) inhibitor, p21 has been shown as a target gene regulated by COM1 during doxobubicin induced chemoresistence (17). In line with this finding, an upregulation of p21 was seen in the bladder cancer cells of COM1 overexpression. The role of p21 has been well studied in mediating the G1/S checkpoint in response to treatment with agents the induce genotoxic stress $(20,21)$. It has been suggested that as a negative regulator of cell cycle progression, $\mathrm{P} 21$ has a certain role to play in the inhibitory effect on growth by COM1. Recently, it has been shown that COM1 is one of the key modulators in the antitumour (pro-apoptotic) effects of the cannabinoids, by mediating its apoptotic effect via upregulation of the endoplasmic reticulum stress related genes ATF-4, CHOP and TRB3 (22). COM1 may also exert its inhibitory effect on prostate cancer via the peroxisome proliferator activated receptor $\gamma$ pathway (23). Together with the recent report that COM1 interacts with p53, a well established tumour suppressor in certain tumour types (15), the present study indicates that regulation of cell cycle and tumour suppressors is a key mechanism in its role in cancer progression. Further investigations would be required to elucidate the molecular mechanisms underlying these impacts on bladder cancer cells by COM1, including the candidate downstream responsive genes, MMP-9 and MMP-13 (24). However, one should bear in mind that the tumour suppressive role of COM1 has only been confirmed in certain tumours, whereas in other tumour types including thyroid and pancreatic cancers, COM1 was found to be overexpressed in tumours $(3,4)$. In colorectal cancer, another intriguing example, although COM1 was found to be lost/reduced in tumour tissues compared with normal tissues (25), the molecule is anti-apoptotic and increase the growth of colorectal cancer cell lines in vitro (26). The tumour suppressive or stimulating role of COM1 appears therefore to be dependent upon the type of tumours, and the experimental conditions. It also depends on the cellular location of the protein, in that cytoplasmic or nuclear location of the protein may govern how the COM1 protein acts, clearly an interesting area to pursue in future studies. Finally, the link between COM1, cellular invasion and apoptosis as seen in the present study and recent studies indicates that COM1 may be a player in the regulation of Anoikis $(27,28)$ and that this interesting link warrants further investigation.

In conclusion, the current study showed reduced expression of COM1 in invasive human bladder cancer. Together with our genetic manipulation study which demonstrated an inverse relationship between COM1 expression and the in vitro growth/invasion, it is concluded that COM1 is a negative regulator of the growth and invasion in bladder cancer.

\section{Acknowledgements}

P.D. is a recipient of Cardiff University China Medical Scholarship. The authors wish to thank the Albert Hung Foundation and Cancer Research Wales for supporting the study.

\section{References}

1. Ree AH, Tvermyr M, Engebraaten O, Rooman M, Rosok O, Hovig E, Meza-Zepeda LA, Bruland OS and Fodstad O: Expression of a novel factor in human breast cancer cells with metastatic potential. Cancer Res 59: 4675-4680, 1999.

2. Mallo GV, Fiedler F, Calvo EL, Ortiz EM, Vasseur S, Keim V, Morisset J and Iovanna JL: Cloning and expression of the rat $\mathrm{p} 8$ cDNA, a new gene activated in pancreas during the acute phase of pancreatitis, pancreatic development, and regeneration, and which promotes cellular growth. J Biol Chem 272: 32360-32369, 1997.

3. Su SB, Motoo Y, Iovanna JL, Berthezene P, Xie MJ, Mouri H, Ohtsubo K, Matsubara F and Sawabu N: Overexpression of $\mathrm{p} 8$ is inversely correlated with apoptosis in pancreatic cancer. Clin Cancer Res 7: 1320-1324, 2001.

4. Ito Y, Yoshida H, Motoo Y, et al: Expression and cellular localization of p8 protein in thyroid neoplasms. Cancer Lett 201: 237-244, 2003 .

5. Jiang WG, Watkins G, Douglas-Jones A, Mokbel K, Mansel RE and Fodstad O: Expression of COM-1/P8 in human breast cancer and its relevance to clinical outcome and ER status. Int J Cancer 117: 730-737, 2005. 
6. Jiang WG, Davies G and Fodstad O: COM-1/P8 in oestrogen regulated growth of breast cancer cells, the ER-beta connection. Biochem Biophys Res Commun 330: 253-262, 2005.

7. Jiang WG, Davies G, Martin TA, Kynaston H, Mason MD and Fodstad O: COM-1/p8 acts as a putative tumour suppressor in prostate cancer. Int J Mol Med 18: 981-986, 2006.

8. Clark DW, Mitra A, Fillmore RA, Jiang WG, Samant RS Fodstad $\mathrm{O}$ and Shevde LA: NUPR1 interacts with p53, transcriptionally regulates p 21 and rescues breast epithelial cells from doxorubicin-induced genotoxic stress. Curr Cancer Drug Targets 8: 421-430, 2008.

9. Allred DC, Harvey JM, Berardo M and Clark GM: Prognostic and predictive factors in breast cancer by immunohistochemical analysis. Mod Pathol 11: 155-168, 1998.

10. Yu H, Ye L, Mansel RE, Zhang Y and Jiang WG: Clinical implications of the influence of Ehm2 on the aggressiveness of breast cancer cells through regulation of matrix metalloproteinase-9 expression. Mol Cancer Res 8: 1501-1512, 2010.

11. Ploeg M, Aben KK and Kiemeney LA: The present and future burden of urinary bladder cancer in the world. World J Urol 27 289-293, 2009.

12. Youssef RF and Lotan Y: Predictors of outcome of non-muscleinvasive and muscle-invasive bladder cancer. Scientific World J 11: 369-381, 2011.

13. Ree AH,Pacheco MM, Tvermyr M, Fodstad O and Brentani MM: Expression of a novel factor, com1, in early tumor progression of breast cancer. Clin Cancer Res 6: 1778-1783, 2000.

14. Ito $\mathrm{Y}$, Yoshida $\mathrm{H}$, Motoo $\mathrm{Y}$, et al: Expression of $\mathrm{p} 8$ protein in medullary thyroid carcinoma. Anticancer Res 25: 3419-3423, 2005.

15. Ree AH, Bratland A, Kroes RA, Aasheim HC, Florenes VA, Moskal JR, Fodstad O, Bruland OS and Maelandsmo GM: Clinical and cell line specific expression profiles of a human gene identified in experimental central nervous system metastases. Anticancer Res 22: 1949-1957, 2002.

16. Giroux V, Malicet C, Barthet M, Gironella M, Archange C Dagorn JC, Vasseur S and Iovanna JL: p8 is a new target of gemcitabine in pancreatic cancer cells. Clin Cancer Res 12 235-241, 2006.
17. Su SB, Motoo Y, Iovanna JL, et al: Expression of $\mathrm{p} 8$ in human pancreatic cancer. Clin Cancer Res 7: 309-313, 2001.

18. Bratland A, Risberg K, Maelandsmo GM, et al: Expression of a novel factor, com1, is regulated by 1,25 -dihydroxyvitamin D3 in breast cancer cells. Cancer Res 60: 5578-5583, 2000.

19. Trump DL, Muindi J, Fakih M, Yu WD and Johnson CS Vitamin D compounds: clinical development as cancer therapy and prevention agents. Anticancer Res 26: 2551-2556, 2006.

20. Niculescu AB III, Chen X, Smeets M, Hengst L, Prives C and Reed SI: Effects of p21(Cip1/Waf1) at both the G1/S and the $\mathrm{G} 2 / \mathrm{M}$ cell cycle transitions: $\mathrm{pRb}$ is a critical determinant in blocking DNA replication and in preventing endoreduplication. Mol Cell Biol 18: 629-643, 1998.

21. Smits VA and Medema RH: Checking out the $\mathrm{G}(2) / \mathrm{M}$ transition. Biochim Biophys Acta 1519: 1-12, 2001.

22. Malicet C, Giroux V, Vasseur S, Dagorn JC, Neira JL and Iovanna JL: Regulation of apoptosis by the p8/prothymosin alpha complex. Proc Natl Acad Sci USA 103: 2671-2676, 2006.

23. Jiang WG, Davies G, Kynaston H, Mason MD and Fodstad O: Does the PGC-1/PPARgamma pathway play a role in COM-1/ p8 mediated cell growth inhibition in prostate cancer? Int J Mol Med 18: 1169-1175, 2006.

24. Goruppi S and Ionanna JL: Stress-inducible protein p8 is involved in several physiological and pathological processes. J Biol Chem 285: 1577-1581, 2010.

25. Davies ML, Parr C, Sanders AJ, Fodstad O and Jiang WG: The transcript expression and protein distribution pattern in human colorectal carcinoma reveal a pivotal role of COM-1/p8 as a tumour suppressor. Cancer Genomics Proteomics 7: 75-80, 2010.

26. Li X, Martin TA and Jiang WG: COM-1/p8 acts as a tumour growth enhancer in colorectal cancer cell lines. Anticancer Res 32: 1229-1237, 2012

27. Frisch SM and Francis HL: Disruption of epithelial cell-matrix interactions induces apoptosis. J Cell Biol 124: 619-626, 1994.

28. Shao J, Sheng H, DuBois RN and Beauchamp RD: Oncogeneic Ras-medicated cell growth arrest and apoptosis are associated with increased ubiquitin-dependent cyclin D1 degradation. J Biol Chem 275: 22916-22924, 2000. 